\title{
Formation continue et droits acquis
}

Olivier Kappelera,

Christoph Hänggeli ${ }^{b}$

a membre du Comité central de la FMH

b administrateur responsable du Secrétariat FPPC
Depuis l'entrée en vigueur, le $1^{\text {er }}$ mai 2003 (assurance-accidents) et le $1^{\text {er }}$ janvier 2004 (assurancemaladie), de la structure tarifaire TARMED, vous ne pouvez en principe facturer la plupart des prestations tarifaires que si vous disposez de la valeur intrinsèque correspondante. Par valeur intrinsèque, on entend les titres de spécialiste, formations approfondies et attestations de formation complémentaire réglées par la RFP. Grâce au principe du maintien des droits acquis, les médecins peuvent néanmoins continuer à exercer leur profession dans les mêmes conditions qu'avant l'entrée en vigueur du TARMED. En d'autres termes, cela signifie que tout médecin qui, avant l'entrée en vigueur de la nouvelle structure tarifaire, fournissait des prestations de manière régulière et sans contestation peut continuer à le faire, et ce même s'il n'est pas détenteur du titre de spécialiste ou des attestations de formation exigées.

\section{Recensement et banque de données des valeurs intrinsèques}

Lors du recensement des valeurs intrinsèques de 2003 et 2004, tous les médecins ont eu la possibilité d'indiquer les prestations qu'ils fournissent dans le cadre des droits acquis. La banque de données sur la valeur intrinsèque comprend actuellement 23000 médecins. 18400 d'entre eux ont déclaré des positions tarifaires relevant des droit acquis. Chaque médecin en a indiqué en moyenne 105 - parmi les 4500 positions tarifaires que compte le TARMED - dans son catalogue personnel de valeur intrinsèque. Il en résulte qu'au total, la banque de données comprend environ deux millions de prestations relevant des droits acquis.

\section{Pendant combien de temps puis-je encore facturer des prestations en vertu des droits acquis?}

Le concept de valeur intrinsèque (version 9.0) entré en vigueur en même temps que le TARMED précise, sous la rubrique «Durée et prolongation», que les prestations relevant des droits acquis doivent faire l'objet d'une formation continue séparée. Par «séparée», on entend que la formation continue a lieu indépendamment de la formation

\section{L'essentiel en bref}

Depuis 2001, vous fournissez régulièrement des prestations pour lesquelles vous ne disposez pas du titre de spécialiste exigé par le TARMED et vous souhaitez pouvoir continuer à le faire dans le cadre des droits acquis? Si la réponse est non, cet article ne vous concerne pas et si la réponse est oui, alors vous devez accomplir une formation continue appropriée. Les prestations relevant des droits acquis et la formation continue s'y rapportant pourront être consultées et confirmées en ligne (www.fmh.ch) à partir de la mi-mai 2006. Les informations concernant l'enregistrement vous parviendront par courrier postal. Une fois l'enregistrement effectué, vous pourrez continuer à facturer ces positions tarifaires à la charge de l'assurance-maladie et accidents pour une nouvelle période de 3 ans.

Lors de sa séance du 17 novembre 2005, le Comité central a approuvé le concept à la base du présent article. Sa mise en œuvre a été négociée avec les partenaires tarifaires.

continue régie par la RFC effectuée en lien avec un titre de spécialiste, une formation approfondie ou une attestation de formation complémentaire. Afin que les médecins puissent continuer à facturer des prestations relevant des droits acquis, ils doivent attester avoir accompli cette formation continue supplémentaire d'ici au 31 décembre 2006.

Domaine AA/AM/AI: délai pour l'attestation de la formation continue supplémentaire

Comme mentionné plus haut, la structure tarifaire TARMED est entrée en vigueur le $1^{\text {er }}$ mai 2003 pour les domaines de l'assurance-accidents (AA), de l'assurance militaire (AM) et de l'assuranceinvalidité (AI). Par conséquent, la formation continue devrait avoir été attestée d'ici au 30 avril 2006. Etant donné qu'il se serait avéré difficile d'imposer différents délais pour l'application du concept de valeur intrinsèque, les assureurs AA/AM/AI ont décidé de faire un geste envers les médecins et se sont déclarés prêts à 
fixer un délai uniforme (31 décembre 2006), synchronisé avec celui des domaines de l'assurancemaladie et de l'ambulatoire hospitalier.

\section{Quelle formation continue dois-je accomplir? Principe de la responsabilité individuelle}

Avec 2 millions de prestations relevant des droits acquis comprenant d'innombrables combinaisons possibles et se rapportant à 18400 médecins, il est tout bonnement impossible de réglementer le type et l'ampleur de la formation continue individuelle à accomplir. Le Comité central, à la demande de la Commission pour la formation postgraduée et continue (CFPC), a donc fixé, le 22 avril 2004 déjà, les modalités de contrôle de la formation continue nécessaire à la facturation des prestations relevant des droits acquis. Il en résulte que l'étendue et la manière dont la formation continue exigée sera accomplie relève de la responsabilité individuelle de chaque médecin. Les paramètres suivants servent de lignes directrices:

- normes en vigueur dans la discipline médicale concernée;

- besoins personnels en formation continue;

- potentiel de risque de la prestation fournie.

La plupart des sociétés de discipline médicale ont transmis des recommandations à leurs membres concernant la fréquentation des sessions de formation continue. Les différentes sociétés de médecine de premier recours regroupées au sein du Collège de médecine de premier recours (CMPR) ont notamment élaboré, pour les principales disciplines concernées, des modules de formation présentés lors des congrès annuels des différentes sociétés. Toutes les sessions de formation continue reconnues par la FMH peuvent être consultées sur le site internet de notre association à la rubrique «Formation continue» (www.fmh.ch).

\section{Existe-t-il un système de contrôle?} Principe de la déclaration volontaire

L'impossibilité de réglementer des milliers de combinaisons différentes en ce qui concerne les droits acquis a entraîné la mise en place du seul système de contrôle envisageable, à savoir la déclaration volontaire à l'instar du droit fiscal par exemple. Dès le mois de mai 2006, vous aurez la possibilité de confirmer par internet que vous avez accompli la formation continue nécessaire pour les prestations que vous souhaitez continuer à facturer en vertu des droits acquis. Le formulaire en ligne comprendra toutes les positions tarifaires que vous avez mentionnées sous les droits acquis dans le cadre du recensement des valeurs intrinsèques. Vous pourrez indiquer quelles positions vous souhaitez continuer à facturer et confirmer que vous avez suivi sous votre propre responsabilité une formation continue dans ces domaines, et que vous êtes en mesure de fournir ces prestations de manière irréprochable sur le plan de la qualité. Cette confirmation de la formation continue accomplie ne concernera pas des prestations individuelles mais s'effectuera de façon globale (cf. annexe). Les informations concernant l'enregistrement en vue de l'accès à vos données de valeur intrinsèque vous parviendront par courrier postal.

\section{Que se passe-t-il si je ne dépose pas} mon attestation de formation continue?

Toutes les données de valeur intrinsèque qui seront modifiées à partir du deuxième trimestre 2006, y compris les attestations de formation continue déposées, permettront la mise à jour de la banque de données sur la valeur intrinsèque. Elles entraîneront vraisemblablement une réduction des positions tarifaires relevant des droits acquis, car on peut supposer que les médecins ayant participé à l'époque au recensement des valeurs intrinsèques y avaient indiqué un certain nombre de prestations «en réserve». Dès le $1^{\text {er }}$ janvier 2007, la banque de données sur la valeur intrinsèque servira de base pour le remboursement des prestations par les assureurs. Tout médecin qui ne sera pas détenteur du titre de spécialiste, de la formation approfondie ou de l'attestation de formation complémentaire exigés ni ne disposera de droits acquis valables, ne pourra plus exiger le remboursement de ces prestations par les caisses-maladie.

\section{Le délai pour la remise de l'attestation de formation continue est-il limité?}

Il n'y a pas d'échéance quant à la remise de l'attestation de formation continue, mais il convient de souligner les conséquences suivantes:

- Quiconque ne rend pas sa déclaration de formation continue d'ici au 31 décembre 2006 doit s'attendre à ce que les prestations qu'il fournit ne soient plus remboursées par les caisses-maladie.

- L'attestation de la formation continue en vue du maintien des droits acquis doit être renouvelée tous les trois ans.

\section{Qu'en est-il des détenteurs du titre} postgrade fédéral de médecin praticien? Et que doivent faire les médecins qui n'ont pas de titre de spécialiste?

Conformément à l'art. 12 de la Réglementation pour la formation continue (RFC), ces médecins 
se conforment au programme de formation continue correspondant à leur activité principale. Dans leur cas, la formation continue est attestée par le diplôme de formation continue décerné par la société de discipline médicale concernée.

\section{Que se passe-t-il si ma déclaration volon-} taire n'est pas conforme à la réalité?

Des discussions sont actuellement en cours avec les assureurs. Dans des cas particuliers, ceux-ci peuvent exiger que le médecin précise et motive la formation continue accomplie et mentionnée dans sa déclaration volontaire. Si la Commission paritaire pour le contrôle de la banque de données sur la valeur intrinsèque (PaKoDig), constituée de représentants du corps médical et des assureurs, aboutit à la conclusion que les précisions et motifs indiqués sont insuffisants, le médecin incriminé bénéficie d'un délai supplémentaire pour attester sa formation continue. S'il ne respecte pas ce délai, la position tarifaire facturée est supprimée des droits acquis et ne sera plus remboursée.

Remarque: le formulaire en ligne permettant d'attester votre formation continue fait office de document officiel. De ce fait, s'il comporte des informations erronées, cela peut entraîner des mesures pénales pour faux dans les titres.

\section{Perspectives}

A partir du $1^{\text {er }}$ janvier 2007, les choses deviennent «sérieuses». En effet, les assureurs ne rem-

Figure 1

Page d'accueil.

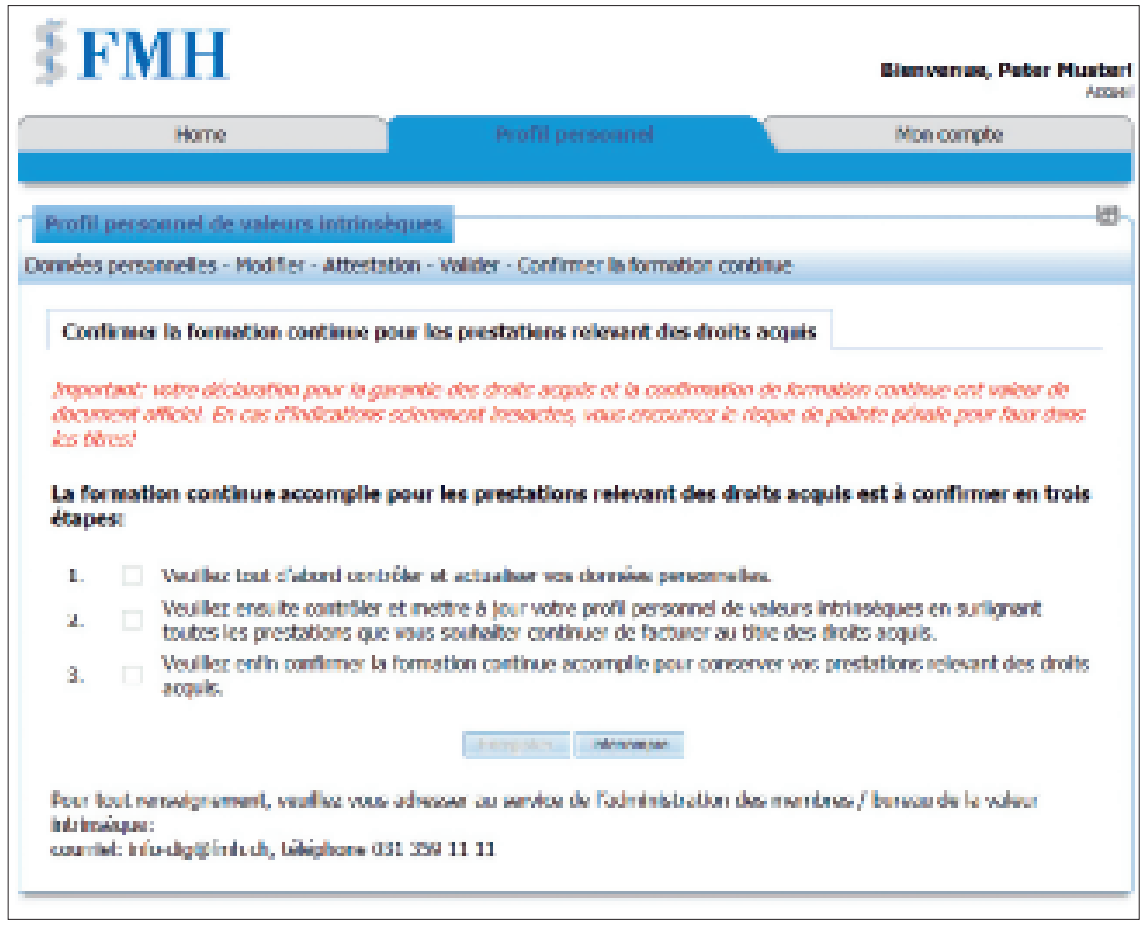

bourseront plus aucune prestation relevant des droits acquis si la formation continue correspondante n'est pas attestée. Au terme de la première année sous ce régime, il sera possible d'évaluer quelles positions tarifaires sont réellement facturées. Les modalités de formation continue et les mesures de contrôle dépendront des résultats de cette analyse.

- Pour les positions tarifaires facturées régulièrement par de nombreux médecins détenteurs du même titre de spécialiste, il conviendra de se demander s'il ne serait pas judicieux d'intégrer cette prestation dans le programme de formation postgraduée correspondant, ce qui rendrait caduque la question des droits acquis.

- Pour les prestations engendrant des coûts élevés ou les prestations à haut risque, il sera peut-être envisageable de prévoir des dispositions spéciales en matière de formation continue.

- Pour les positions tarifaires inutilisées, il conviendra éventuellement de prévoir une date d'échéance.

Ces réglementations spéciales mises à part, il est prévu que l'attestation de la formation continue pour la facturation des prestations relevant des droits acquis devra être renouvelée tous les trois ans.

\section{Glossaire}

Banque de données sur la valeur intrinsèque La banque de données sur la valeur intrinsèque permet à la FMH de gérer l'ensemble des données fournies par les médecins lors du recensement des valeurs intrinsèques.

\section{Catalogue des valeurs intrinsèques} (profil personnel des valeurs intrinsèques)

Le catalogue des valeurs intrinsèques fournit le profil personnel d'un médecin: titres de spécialiste, formations approfondies ou attestations de formation complémentaire et positions tarifaires relevant des droits acquis applicables.

\section{Certificat de facturation}

Tout médecin qui n'est pas porteur d'un titre de spécialiste ou seulement du titre postgrade fédéral de médecin praticien doit, conformément au concept de valeur intrinsèque, s'adjoindre à un titre de spécialiste. Toutes les prestations inhérentes à ce titre sont considérées comme relevant des droits acquis. 
Figure 2

Modification du profil des valeurs intrinsèques.

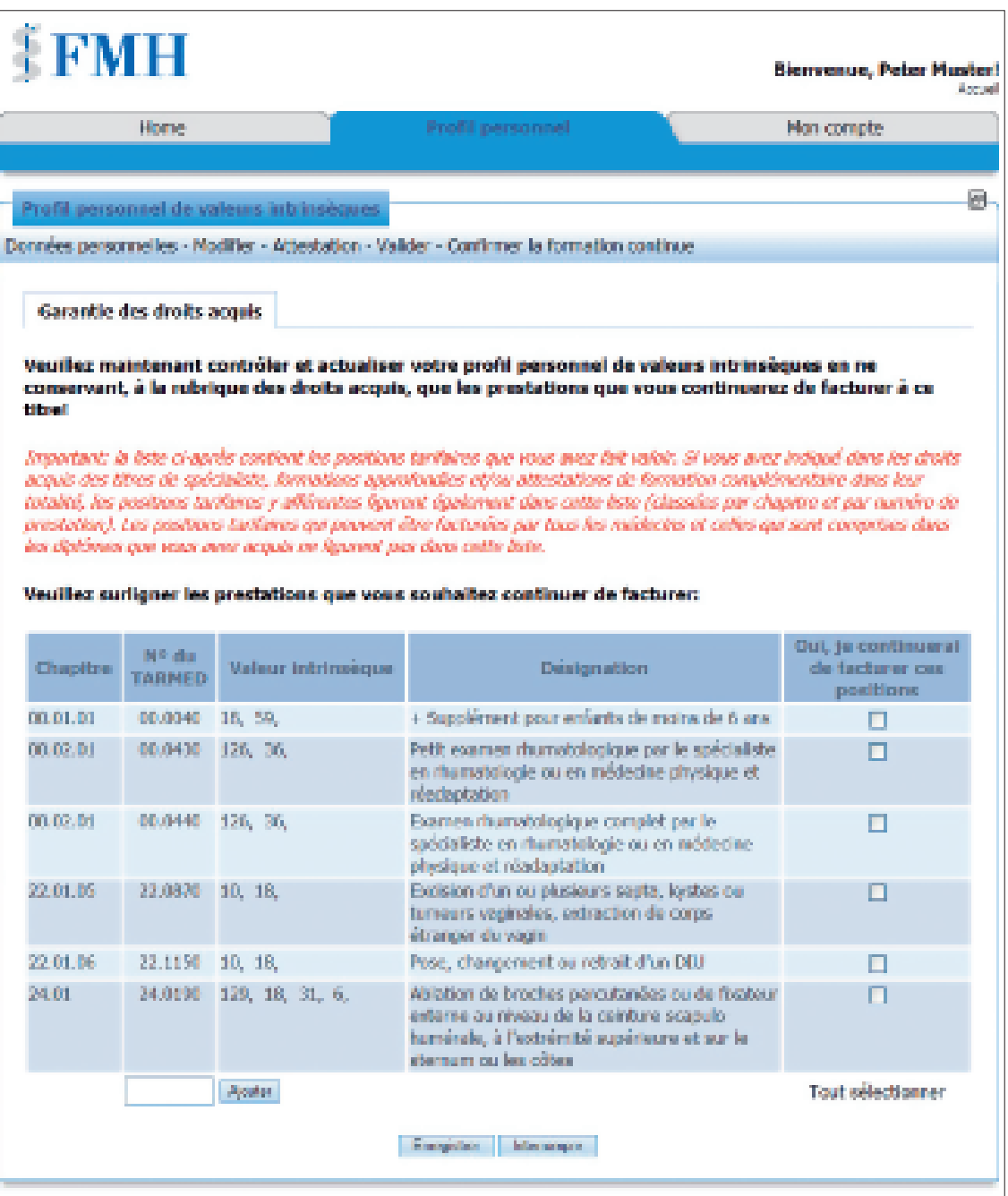

Figure 3

Saisie de l'attestation de formation continue.

\section{\$ FMH}

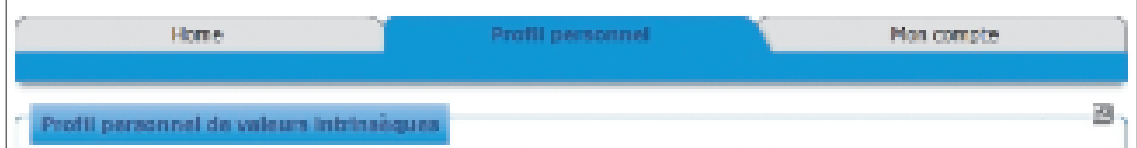

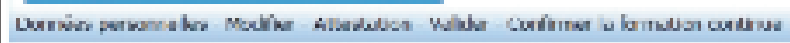

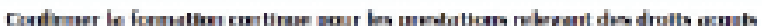

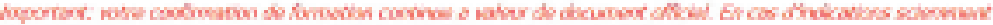

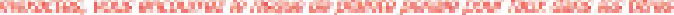

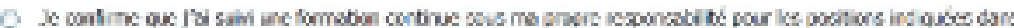

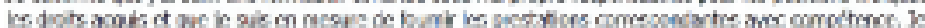

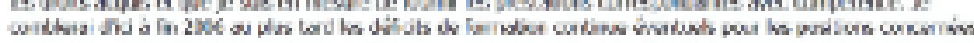

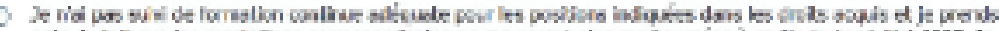

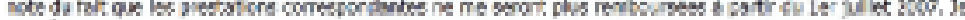

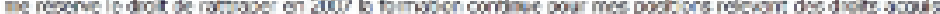

Concept de valeur intrinsèque (version 9.0) Le concept de valeur intrinsèque 9.0 comprend toutes les réglementations passées entre fournisseurs de prestations et assureurs en matière de droit à la facturation des prestations conformément à la nouvelle structure tarifaire TARMED.

\section{PaKoDig}

La commission paritaire composée de représentants du corps médical et des assureurs est chargée du contrôle de la banque de données sur la valeur intrinsèque.

\section{Positions/prestations tarifaires relevant des droits acquis}

Tout médecin qui, avant le $1^{\text {er }}$ janvier 2004, a fourni régulièrement et sans contestation pendant trois ans des prestations peut les inscrire dans son catalogue des droits acquis et continuer à les facturer même s'il n'est pas détenteur du titre correspondant.

\section{Recensement des valeurs intrinsèques}

Dans les années 2003/2004, la FMH a effectué auprès des médecins un recensement de toutes les données de valeur intrinsèque qualitative et leur a demandé quelles prestations ils souhaitaient facturer dans le cadre des droits acquis.

\section{Réglementation pour la formation continue (RFC)}

La Réglementation pour la formation continue de la FMH règle le domaine d'application et les principes de base de la formation continue médicale en Suisse.

\section{Réglementation pour la formation postgraduée (RFP)}

La Réglementation pour la formation postgraduée de la FMH est un ensemble de règles, accréditées par le Département fédéral de l'intérieur (DFI) qui, en complément à la loi sur l'exercice des professions médicales, régit les principes de base de la formation postgraduée médicale et les conditions nécessaires à l'obtention des titres postgrades.

\section{Valeur intrinsèque qualitative}

La valeur intrinsèque qualitative indique quelles qualifications professionnelles (titre de spécialiste, formation approfondie, attestation de formation complémentaire) sont nécessaires, en vertu de la Réglementation pour la formation postgraduée, pour facturer des prestations spécifiques à la charge de l'assurance sociale. La valeur intrinsèque qualitative de chaque prestation figure dans le TARMED. 


\section{Annexe}

Exemple d'attestation de formation continue pour la facturation des prestations relevant des droits acquis

L'attestation de la formation continue pour la facturation des prestations relevant des droits acquis se fait via internet (cf. page d'accueil, fig. 1). La démarche comprend deux étapes:

1. mise à jour du profil personnel de valeur intrinsèque. Indication par le médecin de toutes les prestations relevant des droits acquis qu'il souhaite continuer à facturer après 2006 (fig. 2);

2. saisie de l'attestation de formation continue (fig. 3).
La banque de données sur la valeur intrinsèque: un modèle bientôt dépassé? Pour combien de temps les prestations relevant des droits acquis existeront-elles encore? Par définition et de par leur nature, elles sont transitoires. Leur validité expirera au plus tard dès que tous les médecins autorisés à les facturer auront cessé leur activité. Autrement dit, la problématique des droits acquis s'amenuisera au fil des ans. La formation continue exigée en vue du maintien des droits acquis et son transfert dans un programme de formation postgraduée va encore accélérer la tendance. La suppression des droits acquis rendra inutile le maintien d'une banque de données spéciale sur la valeur intrinsèque. Les détenteurs de titres de spécialiste, de formations approfondies et d'attestations de formation complémentaire figurent en effet d'ores et déjà dans le registre des médecins (www.fmh-index.ch). 